\title{
Superlattice properties of carbon nanotubes in a transverse electric field
}

\author{
O. V. Kibis* \\ Department of Applied and Theoretical Physics, Novosibirsk State Technical University, Novosibirsk 630092, Russia
}

D. G. W. Parfitt and M. E. Portnoi

School of Physics, University of Exeter, Stocker Road, Exeter EX4 4QL, United Kingdom

(Received 7 October 2004; revised manuscript received 26 October 2004; published 19 January 2005)

\begin{abstract}
Electron motion in a $(n, 1)$ carbon nanotube is shown to correspond to a de Broglie wave propagating along a helical line on the nanotube wall. This helical motion leads to periodicity of the electron potential energy in the presence of an electric field normal to the nanotube axis. The period of this potential is proportional to the nanotube radius and is greater than the interatomic distance in the nanotube. As a result, the behavior of an electron in a $(n, 1)$ nanotube subject to a transverse electric field is similar to that in a semiconductor superlattice. In particular, Bragg scattering of electrons from the long-range periodic potential results in the opening of gaps in the energy spectrum of the nanotube. Modification of the band structure is shown to be significant for experimentally attainable electric fields, which raises the possibility of applying this effect to nanoelectronic devices.
\end{abstract}

DOI: 10.1103/PhysRevB.71.035411

\section{INTRODUCTION}

Carbon nanotubes (CNTs) are cylindrical molecules with nanometer diameter and micrometer length. Since the discovery of CNTs just over a decade ago, ${ }^{1}$ their unique electronic and structural properties have aroused great excitement in the scientific community and promise a broad range of applications. Significant theoretical effort has been applied to develop refined models of the electronic structure of carbon nanotubes, as well as their optical and transport properties, although even a simple tight-binding model ${ }^{2}$ yielding analytic solutions is sufficient to elucidate key nanotube features (e.g., whether a CNT of given structure will exhibit metallic or semiconducting properties). In this paper we apply such a model to a particular type of single-wall CNT, a so-called $(n, 1)$ nanotube. In Sec. II we show that for such a CNT the electron motion corresponds to a de Broglie wave propagating along a helical line. The theoretical treatment of this type of CNT in an electric field perpendicular to the nanotube axis (transverse electric field) can be reduced to a one-dimensional superlattice problem (see Sec. III). Such superlattice behavior of current-carrying electrons suggests the application of CNTs to the development of carbon nanotubebased devices.

\section{ENERGY SPECTRUM OF $(n, 1)$ NANOTUBES}

A single-wall carbon nanotube may be considered as a single graphite sheet rolled into a cylinder. The electronic energy spectrum of the CNT is therefore intimately related to the energy spectrum $\varepsilon_{g 2 \mathrm{D}}(\mathbf{k})$ of a two-dimensional (2D) graphite sheet, which can be written in the tight-binding approximation as $^{2}$

$$
\varepsilon_{g 2 \mathrm{D}}(\mathbf{k})= \pm \gamma_{0}\left|\exp \left(\frac{i k_{x} a}{\sqrt{3}}\right)+2 \exp \left(-\frac{i k_{x} a}{2 \sqrt{3}}\right) \cos \left(\frac{k_{y} a}{2}\right)\right|
$$

where $k_{x}$ and $k_{y}$ are the electron wave vector components in the graphite sheet plane along the $x$ and $y$ axes, respectively
PACS number(s): 73.22.-f, 73.63.Fg, 78.67.Pt

(see Fig. 1). In the energy spectrum (1), the plus and minus signs correspond to the conduction and valence bands, respectively, $\gamma_{0} \approx 3 \mathrm{eV}$ is the transfer integral between $\pi$-orbitals of neighboring carbon atoms, and the lattice constant $a=\left|\mathbf{a}_{1}\right|=\left|\mathbf{a}_{2}\right|=\sqrt{3} \times a_{c-c}=2.46 \AA$, where $\mathbf{a}_{1}$ and $\mathbf{a}_{2}$ are the $2 \mathrm{D}$ basis vectors and $a_{c-c}=1.42 \AA$ is the interatomic distance in graphite. The way in which the $2 \mathrm{D}$ graphite sheet is rolled up to form the CNT can be described by two vectors, the translation vector $\mathbf{T}$ and the chiral vector $\mathbf{C}_{h}$ (see Fig. 1). The chiral vector $\mathbf{C}_{h}$ can be expressed in terms of the $2 \mathrm{D}$ basis vectors of the unrolled graphite sheet as $\mathbf{C}_{h}=n \mathbf{a}_{1}$ $+m \mathbf{a}_{2}$, where the pair of integers $(n, m)$ is used as a standard notation $^{2}$ for a CNT of given crystal structure. To obtain the electronic energy spectrum of the $(n, m) \mathrm{CNT}$, we begin by expressing the wave vector $\mathbf{k}$ in terms of components along $\mathbf{T}$ and $\mathbf{C}_{h}$ as $\mathbf{k}=k_{\|} \mathbf{T} / T+k_{\perp} \mathbf{C}_{h} / C_{h}$, where $k_{\|}$and $k_{\perp}$ are subject to the following constraints: $-\pi / T<k_{\|} \leqslant \pi / T$ and $k_{\perp}$ $=2 \pi l / C_{h}(l=0,1,2, \ldots, N-1)$. The integer $l$ represents the electron angular momentum along the nanotube axis and

$$
N=\frac{2\left(n^{2}+m^{2}+n m\right)}{d_{R}},
$$

is the number of elementary atomic cells consisting of two carbon atoms $(A, B)$ per area $\left|\mathbf{C}_{h} \times \mathbf{T}\right|$. The number $d_{R}$ appearing in Eq. (2) is the greatest common divisor of the two integers $(2 n+m, 2 m+n)$. The lengths of the chiral vector and translation vector are given by $C_{h}=a \sqrt{n^{2}+m^{2}+n m}$ and $T$ $=\sqrt{3} C_{h} / d_{R}$, respectively.

The energy spectrum of a $(n, m)$ CNT can be obtained by expressing $k_{x}$ and $k_{y}$ in terms of $k_{\|}$and $k_{\perp}$, and substituting them in Eq. (1), thus yielding

$$
\varepsilon= \pm \gamma_{0}\left|\exp \left[\frac{i \sqrt{3} a}{2}\left(k_{\|} \cos \theta-k_{\perp} \sin \theta\right)\right]+2 \cos \left(\frac{k_{s} a}{2}\right)\right|,
$$

where we have introduced the new parameter $k_{s}=k_{\perp} \cos \theta$ $+k_{\|} \sin \theta$, and the chiral angle $\theta(|\theta| \leqslant \pi / 6)$ shown in Fig. 1 . Taking into account that 


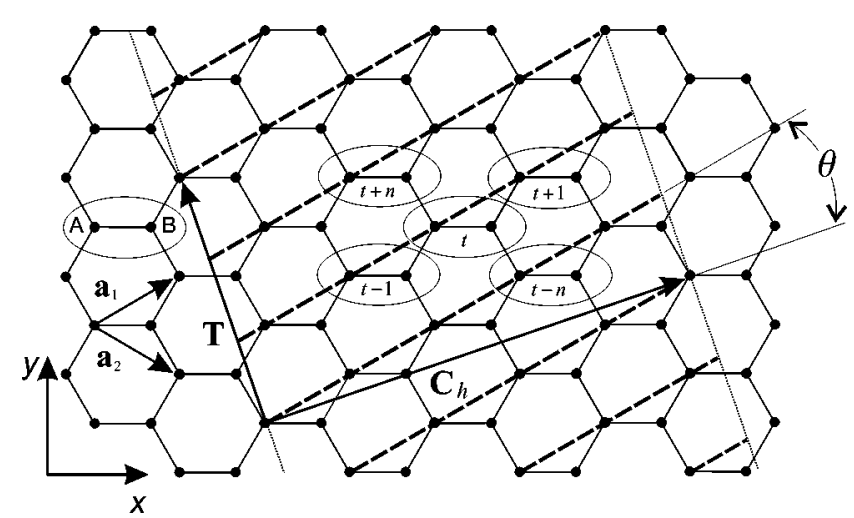

FIG. 1. The unrolled graphite sheet. By connecting the head and tail of the chiral vector $\mathbf{C}_{h}$ we can construct, for example, a $(4,1)$ carbon nanotube. The dashed lines will then form a helical line on the nanotube wall.

$$
\cos \theta=\frac{2 n+m}{2 \sqrt{n^{2}+m^{2}+n m}}, \quad \sin \theta=\frac{\sqrt{3} m}{2 \sqrt{n^{2}+m^{2}+n m}}
$$

we have, for $m \neq 0$, the equation

$$
\sqrt{3}\left(k_{\|} \cos \theta-k_{\perp} \sin \theta\right) a=\left[(2 n+m) k_{s} a-2 k_{\perp} C_{h}\right] / m .
$$

Substituting Eqs. (4) and (5) into Eq. (3) we obtain

$$
\varepsilon= \pm \gamma_{0}\left|\exp \left[i\left(\frac{2 n+m}{2 m} k_{s} a-\frac{k_{\perp} C_{h}}{m}\right)\right]+2 \cos \left(\frac{k_{s} a}{2}\right)\right|
$$

which, together with the constraint $k_{\perp}=2 \pi l / C_{h}$, yields an electron energy spectrum of the form

$$
\begin{aligned}
\varepsilon= & \pm \gamma_{0}\left[1+4 \cos \left(\frac{k_{s} a}{2}\right) \cos \left(\frac{2 n+m}{2 m} k_{s} a-\frac{2 \pi l}{m}\right)\right. \\
& \left.+4 \cos ^{2}\left(\frac{k_{s} a}{2}\right)\right]^{1 / 2} .
\end{aligned}
$$

For $m=1$, Eq. (7) becomes independent of $l$, and we obtain the electron energy spectrum of a $(n, 1) \mathrm{CNT}$ in the form

$\varepsilon_{j}\left(k_{s}\right)=(-1)^{j} \gamma_{0}\left[1+8 \cos \left(\frac{n+1}{2} k_{s} a\right) \cos \left(\frac{n k_{s} a}{2}\right) \cos \left(\frac{k_{s} a}{2}\right)\right]^{1 / 2}$,

where $j=1,2$ correspond to the valence and conduction bands, respectively. It should be noted that the spectrum (8) depends on the parameter $k_{s}$ alone, in contrast to the general case of a $(n, m) \mathrm{CNT}$, for which the electron energy spectrum depends on two parameters $\left(k_{\|}\right.$and $k_{\perp}$ are conventionally used). This peculiarity of a $(n, 1) \mathrm{CNT}$ is a consequence of its special crystal symmetry: the $(n, 1)$ CNT lattice can be obtained by translation of an elementary two-atom cell along a helical line on the nanotube wall (see Fig. 1). As a result, the parameter $k_{s}$ has the meaning of an electron wave vector along the helical line, and so any possible electron motion in a $(n, 1)$ CNT can be described by a de Broglie wave propagating along such a line. Thus, $(n, 1)$ CNTs represent a previously overlooked distinctive class of nanotubes, which

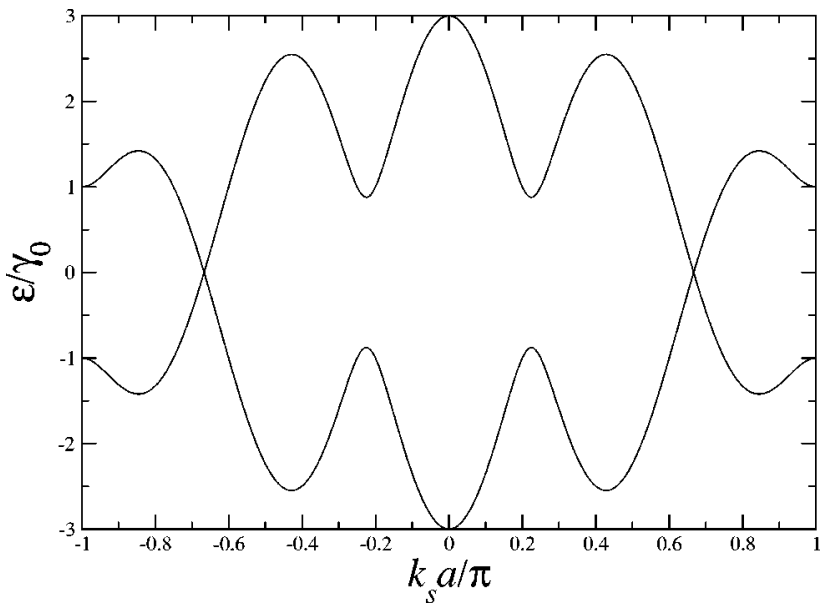

FIG. 2. Electron energy spectrum of a metallic $(4,1) \mathrm{CNT}$ as a function of the wave number $k_{s}$ along a helical line on the nanotube wall.

may be termed helical nanotubes. The electron energy spectrum of a $(4,1)$ CNT as a function of the helical wave number $k_{s}$ is shown in Fig. 2. The band gap for this natotube closes at $k_{s} a=2 \pi / 3$, and it can be shown that the same is true for all metallic $(n, 1)$ nanotubes.

\section{HELICAL NANOTUBES IN A TRANSVERSE ELECTRIC FIELD}

Both descriptions of the energy spectrum of a $(n, 1)$ CNT-by two parameters, $k_{\|}$and $k_{\perp}$, or a single parameter $k_{s}$ - are physically equivalent. However, the second description is more convenient for studies of electron processes determined by the above-mentioned helical symmetry of electron motion, and allows one to discover new physical effects (e.g., the electron-electron interaction should be strongly modified for helical one-dimensional motion ${ }^{3}$ ). We shall now show that such helical symmetry results in superlattice behavior of a $(n, 1) \mathrm{CNT}$ in the presence of an electric field oriented perpendicular to the nanotube axis (a transverse electric field).

The potential energy of an electron on a helix subject to a transverse electric field takes the form

$$
U=e E R \cos \left(\frac{2 \pi s}{l_{0}}\right)
$$

where $e$ is the electron charge, $E$ is the electric field strength, $R=C_{h} / 2 \pi$ is the radius of the CNT, $s$ is the electron coordinate along the above-mentioned helical line,

$$
l_{0}=\frac{2 \pi R}{\cos \theta}=\frac{2 a\left(n^{2}+n+1\right)}{2 n+1},
$$

is the length of a single coil of the helix, and the electric potential is assumed to be zero at the axis of the CNT. The potential energy (9) is periodic in the electron coordinate $s$ along the helical line and the period of the potential is equal to $l_{0}$. Since this period (10) is proportional to the CNT radius $R$ and is greater than the interatomic distance $a_{c-c}$, the CNT 
assumes typical superlattice properties. In particular, Bragg reflection of electron waves with wave vectors $k_{s}= \pm \pi / l_{0}$ results in energy splitting within the conduction and valence bands of the CNT. We shall now study this effect in more detail.

In the framework of the tight-binding model, ${ }^{2}$ considering only three nearest neighbors to each atom, the wave functions for electron states with corresponding energies (8) can be written as

$$
\psi_{j}\left(k_{s}\right)=\frac{1}{\sqrt{2 M}} \sum_{t}\left[\psi_{t}^{(A)}+(-1)^{j} \frac{h *\left(k_{s}\right)}{\left|h\left(k_{s}\right)\right|} \psi_{t}^{(B)}\right] \exp \left(i k_{s} t a\right),
$$

where $M$ is the total number of two-atom cells in the CNT, $\psi_{t}^{(A)}$ and $\psi_{t}^{(B)}$ are $\pi$-orbital wave functions for the two carbon atoms $A$ and $B$, respectively, $t$ is the number along the helical line for an elementary cell consisting of these two atoms (see Fig. 1), and $h\left(k_{s}\right)=1+\exp \left(-i k_{s} a\right)+\exp \left(i n k_{s} a\right)$. The value of the potential energy $U$ in the external electric field at the position of a particular atom of the CNT depends on the angle between the electric field vector and the vector normal to the nanotube axis which passes through this atom. As a consequence, the coordinate of atom $A$ in cell number $t$ along the helical line is

$$
s=a t+\frac{l_{0}}{2 \pi} \phi .
$$

The angle $\phi$ is defined in such a way that $R \cos [\phi+\pi(n$ $\left.+1) /\left(n^{2}+n+1\right)\right]$ is the coordinate in the direction of the electric field (with zero at the CNT axis) of atom $B$ in the cell with $t=0$. Using Eqs. (11) and (12), we can write the matrix element of the potential energy (9) as

$$
\begin{aligned}
\left\langle\psi_{i}\left(k_{s}^{\prime}\right)|U| \psi_{j}\left(k_{s}\right)\right\rangle= & V_{i j}^{+} \delta_{\cos \left(k_{s} a-k_{s}^{\prime} a+2 \pi a / l_{0}\right), 1} \\
& +V_{i j}^{-} \delta_{\cos \left(k_{s} a-k_{s}^{\prime} a-2 \pi a / l_{0}\right), 1}
\end{aligned}
$$

where

$$
\begin{aligned}
V_{i j}^{ \pm}= & \frac{e E R}{4}\left[1+\left(2 \delta_{i j}-1\right) \frac{h\left(k_{s}^{\prime}\right) h *\left(k_{s}\right)}{\left|h\left(k_{s}^{\prime}\right) h\left(k_{s}\right)\right|} \exp \left( \pm i \frac{\pi(n+1)}{n^{2}+n+1}\right)\right] \\
& \times \exp ( \pm i \phi),
\end{aligned}
$$

and $\delta_{\alpha \beta}$ is the Kronecker delta. In the derivation of Eqs. (13) and (14) we have also assumed that the external electric field $E$ is much less than the atomic field, i.e.,

$$
E \ll \frac{\gamma_{0}}{e a} .
$$

This allows us to neglect any change in the atomic wave functions $\psi_{t}^{(A)}$ and $\psi_{t}^{(B)}$ due to the field $E$, and we take into account only the mixing of states (11) by the field. According to Eq. (13), the field mixes only electron states (11) with wave vectors differing by $2 \pi / l_{0}$. In this approximation, the exact wave function in the presence of the electric field, $\psi_{E}\left(k_{s}\right)$, can be expressed as a superposition of wave functions (11) with $k_{s}$ shifted by integer numbers of $2 \pi / l_{0}$ :

$$
\psi_{E}\left(k_{s}\right)=\sum_{j=1}^{2} \sum_{\nu=0}^{\mu-1} b_{j \nu} \psi_{j}\left(k_{s}+2 \pi \nu / l_{0}\right)
$$

To ensure that in Eq. (16) we sum only over different electron states, the parameter $\mu$ should be the smallest integer defined by the condition $\psi_{j}\left(k_{s}\right)=\psi_{j}\left(k_{s}+2 \pi \mu / l_{0}\right)$. This condition, together with the $2 \pi / a$ periodicity of $\psi_{j}\left(k_{s}\right)$, implies that $\mu / l_{0}=\beta / a$, where $\beta$ is the smallest integer for which this equality is satisfied. Using Eq. (10) together with Eq. (2) one can obtain $\beta=(2 n+1) / d_{R}$, which yields $\mu=N$. This result has a transparent physical interpretation, since the two closest carbon atoms equivalent with respect to a translation parallel to the nanotube axis are separated by a distance $\mathrm{Na}$ along a helical line.

Substituting the wave function (16) into the Schrödinger equation with the potential energy (9) we obtain a system of equations for the coefficients $b_{j \nu}$ entering Eq. (16),

$$
\begin{aligned}
& {\left[\varepsilon_{j}\left(k_{s}+2 \pi \nu / l_{0}\right)-\varepsilon_{E}\left(k_{s}\right)\right] b_{j \nu}} \\
& \quad+\sum_{i=1}^{2} \sum_{\nu^{\prime}=0}^{N-1}\left\langle\psi_{j}\left(k_{s}+2 \pi \nu / l_{0}\right)|U| \psi_{i}\left(k_{s}+2 \pi \nu^{\prime} / l_{0}\right)\right\rangle b_{i \nu^{\prime}}=0,
\end{aligned}
$$

where $\nu=0,1,2, \ldots, N-1$, the index $j$ takes the value 1 or 2 for the valence and conduction bands, respectively, and $\varepsilon_{E}\left(k_{s}\right)$ is the electron energy in the presence of the transverse electric field.

Let us consider the states $k_{s}=-\pi / l_{0}$ and $\pi / l_{0}$ in the same CNT energy band, which are at the boundaries of a Brillouin zone created by the periodic superlattice potential (9) of the external field. One should expect the appearence of energy gaps at these values of $k_{s}$ due to Bragg reflection of electron waves from the superlattice potential. These states are separated by $2 \pi / l_{0}$ and have the same energy, which means that they are strongly mixed by the electric field. For these values of $k_{s}$ it can be shown that the contributions to the sum in Eq. (16) from all other states can be neglected for sufficiently weak fields, $E \ll \gamma_{0} a /\left(e R^{2}\right)$. As a result, the system of equations (17) is reduced to just two equations, from which the energy of Bragg band splitting $\Delta \varepsilon$ is found to be

$$
\Delta \varepsilon=2\left|\left\langle\psi_{j}\left(-\pi / l_{0}\right)|U| \psi_{j}\left(\pi / l_{0}\right)\right\rangle\right| \sim e E R .
$$

Thus, even a small electric field results in a superlattice-like change of the electron energy spectrum in $(n, 1)$ CNTs, with the appearance of Bragg energy gaps proportional to the field amplitude $E$ and the nanotube radius $R$. Notably, this dependence of the Bragg gaps on the external field and radius applies to any helical quasi-one-dimensional nanostructure in a transverse electric field: this generic feature arises from the symmetry of the nanostructure, and is independent of the parameters of the tight-binding model used to derive Eq. (18). For example, it should be possible to observe a similar effect in recently fabricated $\mathrm{InGaAs} / \mathrm{GaAs}$ and $\mathrm{Si} / \mathrm{SiGe}$ semiconductor nanohelices. ${ }^{4,5}$

It should be emphasized that for single-wall carbon nanotubes the discussed superlattice behavior is a unique feature of $(n, 1)$ structures only. For the general case of a $(n, m)$ CNT 
with $m \neq 1$, the energy spectrum (7) depends on the quantum number $l$ in addition to $k_{s}$. As already mentioned, $l$ represents the projection of the electron angular momentum on the nanotube axis, and it follows from the corresponding selection rule that the transverse electric field only mixes electron states with angular momentum $l$ and $l \pm 1$. For $m \neq 1$, however, states with $l$ differing by one correspond to different subbands, and in general have different energies for $k_{s}$ $= \pm \pi / l_{0}$, so that there is no Bragg scattering between these states. The only effect of the electric field, therefore, is to mix electron states with different energies, which does not lead to noticeable modification of the dispersion curves for weak electric fields. ${ }^{6}$

For the particular case of a $(1,1)$ CNT the energy spectrum can be obtained in analytic form for any electron state, since the system of equations (17) consists of four equations only. This system results in a biquadratic equation for the eigenvalues $\varepsilon_{E}\left(k_{s}\right)$,

$$
\varepsilon_{E}^{4}\left(k_{s}\right)-\varepsilon_{E}^{2}\left(k_{s}\right)\left(w_{1}^{2}+w_{2}^{2}+2 v_{1}+2 v_{2}\right)+\left(v_{2}-v_{1}+w_{1} w_{2}\right)^{2}=0,
$$

where $\quad w_{1}=\gamma_{0}\left[1+2 \cos \left(k_{s} a\right)\right], \quad w_{2}=\gamma_{0}\left[1-2 \cos \left(k_{s} a\right)\right], \quad v_{1}$ $=[V \cos (\phi+\pi / 3)]^{2}, \quad v_{2}=[\sqrt{3} V \sin (\phi+\pi / 3)]^{2}, \quad$ and $\quad V$ $=\sqrt{3} e E a /(4 \pi)$. The energy spectrum $\varepsilon_{E}\left(k_{s}\right)$ obtained from Eq. (19) is shown in Fig. 3 (solid lines) for a range of wave vectors $-\pi / a \leqslant k_{s} \leqslant \pi / a$. In the figure, positive energies correspond to the conduction band and negative energies to the valence band. The energy spectrum in the absence of the field is shown for comparison (dashed lines). According to Eq. (10), the superlattice period $l_{0}$ for a $(1,1)$ CNT is equal to twice the lattice constant $a$. Therefore, as can be seen in Fig. 3 , the width of the first Brillouin zone in the presence of a transverse electric field is half that without the field. It can also be seen that the electric field opens gaps in the dispersion curve at $k_{s}= \pm \pi /(2 a)$ due to the aforementioned Bragg reflection of electron waves. For electric fields satisfying condition (15), we obtain from Eq. (19) the Bragg gap

$$
\Delta \varepsilon=\frac{\sqrt{3} e E a}{2 \pi}|\cos (\phi+\pi / 3)| .
$$

The result in Eq. (20) can also be obtained from the more general formula (18). It should be noted that the Bragg gap, as well as the whole energy spectrum of the CNT in a transverse electric field, depends on the orientation of the CNT relative to the field (i.e., on the angle of rotation $\phi$ ). In particular, when $\phi=\pi / 6$ the Bragg gap (20) is zero: for this angle the values of the electric field potential at atoms $A$ and $B$ in a $(1,1)$ CNT are equal in magnitude but opposite in sign, and so the mean value of the potential within one elementary cell of the CNT is zero.

In the general case of a $(n, 1)$ nanotube, for external electric field intensities attainable in experiment $(E$ $\sim 10^{5} \mathrm{~V} / \mathrm{cm}$ ) and for a typical nanotube of radius $R \sim 10 \AA$, the value of the Bragg gap given by (18) is $\Delta \varepsilon \sim 10^{-2} \mathrm{eV}$, which is comparable to the characteristic energy of band splitting in conventional semiconductor superlattices. As a consequence, the discussed superlattice effects generated by the transverse electric field in $(n, 1)$ CNTs should be observ-

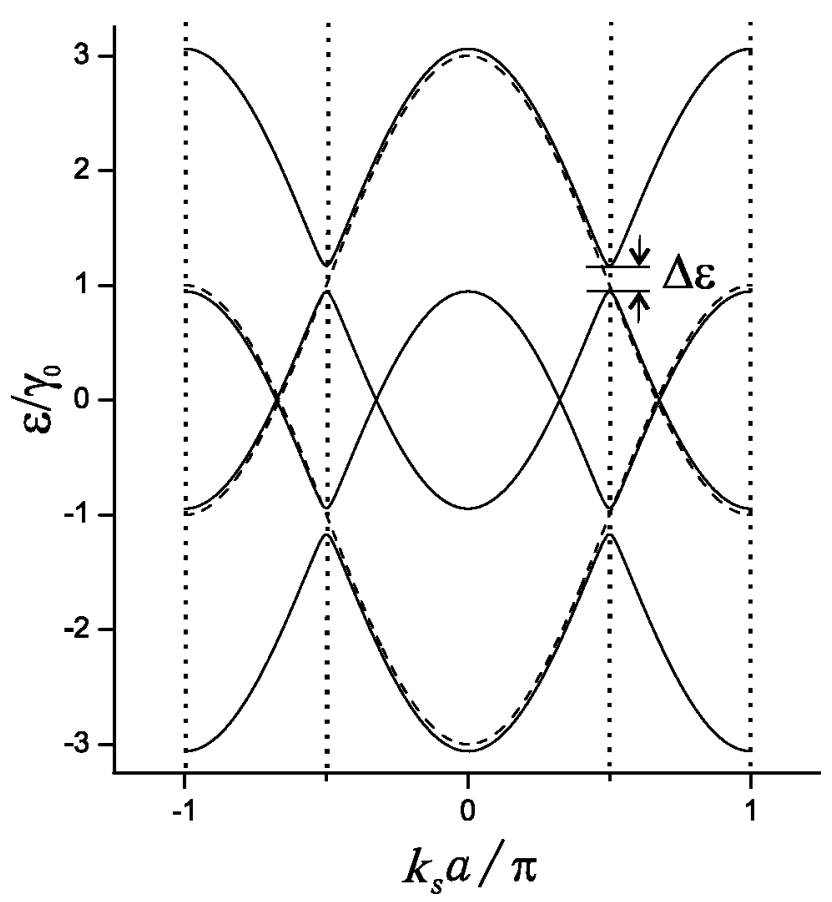

FIG. 3. Electron energy spectrum of a $(1,1) \mathrm{CNT}$ in the presence of a transverse electric field $E=\gamma_{0} /\left(e a_{c-c}\right)$ with $\phi=0$ (solid lines) and without the electric field (dashed lines). The inner pair of vertical dotted lines indicates the first Brillouin zone boundary in the presence of the field, whereas the outer pair corresponds to the first Brillouin zone boundary without the field. $\Delta \varepsilon$ is the Bragg gap opened by the electric field.

able in experiments, and may take place in existing CNT field-effect devices. ${ }^{7}$ The inherent regularity of a nanotubebased superlattice, with the superlattice period determined by the CNT radius, presents a distinct advantage over semiconductor superlattices, in which monolayer fluctuations are unavoidable. A whole range of new nanoelectronic devices based on the discussed superlattice properties of $(n, 1)$ CNTs can be envisaged, including Bloch oscillators ${ }^{8}$ and quantum cascade lasers. $^{9}$ An evaluation of the feasibility of these devices and selection of their optimal parameters will undoubtedly form the subject of extensive future research.

\section{CONCLUSIONS}

In this paper we have discussed a previously overlooked class of CNTs, which may be termed 'helical' nanotubes. While we have concentrated on the superlattice behavior of such nanotubes in a transverse electric field, we also expect their unique symmetry to manifest itself in modification of the electron-electron, electron-phonon, and electron-photon interactions. In addition, we have shown that superlattice behavior in a transverse electric field is a generic feature of helical quasi-one-dimensional nanostructures, which raises new possibilities for developing optoelectronic devices operating in the terahertz range of frequencies.

\section{ACKNOWLEDGMENTS}

This work is supported by the Royal Society, INTAS, the Russian Foundation for Basic Research, and the Russian Universities program. 
*Electronic address: Oleg.Kibis@nstu.ru

${ }^{1}$ S. Iijima, Nature (London) 354, 56 (1991).

${ }^{2}$ R. Saito, G. Dresselhaus, and M. S. Dresselhaus, Physical Properties of Carbon Nanotubes (Imperial College Press, London, 1998).

${ }^{3}$ O. V. Kibis, Phys. Lett. A 166, 393 (1992).

${ }^{4}$ V. Ya. Prinz, V. A. Seleznev, A. K. Gutakovsky, A. V. Chehovskiy, V. V. Preobrazhenskii, M. A. Putyato, and T. A. Gavrilova, Physica E (Amsterdam) 6, 828 (2000).
${ }^{5}$ V. Ya. Prinz, D. Grützmacher, A. Beyer, C. David, B. Ketterer, and E. Deckardt, Nanotechnology 12, 399 (2001).

${ }^{6}$ Y. Li, S. V. Rotkin, and U. Ravaioli, Nano Lett. 3, 183 (2003).

${ }^{7}$ J. Appenzeller, J. Knoch, V. Derycke, R. Martel, S. Wind, and Ph. Avouris, Phys. Rev. Lett. 89, 126801 (2002).

${ }^{8}$ L. Esaki and R. Tsu, IBM J. Res. Dev. 14, 61 (1970).

${ }^{9}$ J. Faist, F. Capasso, D. L. Sivco, C. Sirtori, A. L. Hutchinson, and A. Y. Cho, Science 264, 553 (1994). 